\title{
Acceleration Mechanism in Critical Nucleation of Polymers by Epitaxy of Nucleating Agent
}

\author{
By Tsuyoshi URUSHIHARA,,$^{1, *}$ Kiyoka OKADA, ${ }^{2}$ Kaori WATANABE, ${ }^{2}$
} Akihiko TODA, ${ }^{2}$ Naoshi KAWAMOTO, ${ }^{1}$ and Masamichi HIKOSAKA ${ }^{2}$

Effect of epitaxy of nucleating agent (NA) on nucleation of polymers was investigated based on kinetic study. Nucleation rate $(I)$ of polymers is given by $I=I_{0} \exp \left(-\Delta G^{*} / k T_{\mathrm{c}}\right)(1)$, where $I_{0}$ is prefactor, $\Delta G^{*}$ is free energy for forming critical nucleus, $k$ is Boltzmann constant and $T_{\mathrm{c}}$ is crystallization temperature. We reported in the previous paper that decreasing the size of $\mathrm{NA}$ is important for increasing $I_{0}$, i.e., $I$. In this study we focus on $\Delta G^{*}$ dependence of $I$. In the case of heterogeneous nucleation, $\Delta G^{*}$ is a function of interfacial free energy between NA and nucleus $(\Delta \sigma) . \Delta \sigma / \sigma$ varies between 0 and 1 where $\sigma$ is lateral surface free energy of nucleus. $\Delta \sigma / \sigma$ of isotactic polypropylene (iPP) mixed with three NAs were between 0.13 and 0.23 , which were evaluated by the fit of eq 1 to the experimental data of $I$. $I$ increased by 38 orders of magnitude for $\Delta T=30 \mathrm{~K}$ with decreasing $\Delta \sigma / \sigma$ from 1 to 0 . In the case of NA, $I$ increased by 60 times for $\Delta T=30 \mathrm{~K}$ with decreasing $\Delta \sigma / \sigma$ from 0.23 to 0.13 . I significantly depends on $\Delta \sigma$. Therefore we concluded that $\Delta G^{*}(\Delta \sigma)$ is essentially important for acceleration of nucleation.

KEY WORDS: Nucleating Agent / Epitaxy / Nucleation / Polypropylene /

In our previous study, acceleration mechanism of heterogeneous nucleation by nucleating agent (NA) was investigated based on kinetic study. ${ }^{1,2}$ In the classical nucleation theory (CNT) nucleation rate $I$ is generally expressed as a product of two factors, prefactor $I_{0}$ and probability of forming a critical nucleus, ${ }^{3,4}$

$$
I=I_{0} \exp \left(-\frac{\Delta G^{*}}{k T_{c}}\right)
$$

where $\Delta G^{*}$ is free energy for forming critical nucleus, $k$ is Boltzmann constant and $T_{\mathrm{c}}$ is crystallization temperature.

In our previous studies, ${ }^{1,2}$ acceleration mechanism of the first factor of $I_{0}$ by NA was studied by assuming epitaxy between NA and polymer crystals. The acceleration mechanism by $\Delta G^{*}$ in heterogeneous nucleation has not been studied based on kinetic study. Purpose of this study is to clarify the important role of $\Delta G^{*}$ in acceleration mechanism of heterogeneous nucleation.

$I_{0}$ was predicted in our previous study by

$$
I_{0} \propto C_{\mathrm{NA}} a_{\mathrm{NA}}^{-1},
$$

where $C_{\mathrm{NA}}$ is concentration of NA in the mixture of NA and polymer and $a_{\mathrm{NA}}$ is the largest lateral size of a NA crystal. ${ }^{1}$ Eq 2 was confirmed experimentally in our previous study. ${ }^{1,2}$ It is concluded that $I_{0}$ is accelerated by significant epitaxy between NA and iPP crystals.

\section{Role of $\Delta G^{*}(\Delta \sigma)$ in Acceleration of Heterogeneous Nucle- ation}

In this study, we will focus on the second factor of eq 1 , $\Delta G^{*}{ }^{3,4}$ When we assume rectangular parallelepiped nucleus on the basis of $\mathrm{CNT},{ }^{5} \Delta G$ of heterogeneous nucleation is given by

$$
\Delta G=-\operatorname{lm} n \Delta g+2 \operatorname{lm} \sigma+2 m n \sigma_{e}+\ln \Delta \sigma
$$

where $l, m$ and $n$ are the length of each edge of a nucleus, $\Delta g$ is the bulk free energy of fusion, $\sigma$ and $\sigma_{\mathrm{e}}$ are lateral and end surface free energy, respectively, and $\Delta \sigma$ is defined by

$$
\Delta \sigma \equiv \sigma+\sigma_{0 s}-\sigma_{s},
$$

where $\sigma_{0 \mathrm{~s}}$ and $\sigma_{\mathrm{s}}$ are the interfacial energy between nucleus and heterogeneity and the surface free energy of heterogeneity, respectively. In this study, $l, m, n$ are counted by repeating unit, and $\Delta g, \sigma, \sigma_{\mathrm{e}}, \sigma_{0 \mathrm{~s}}, \sigma_{\mathrm{s}}$, and $\Delta \sigma$ are defined per repeating unit. Eq 3 represents hypersurface, and the position of extremum designates $\Delta G^{*}$. The positions of $l, m$ and $n$ with respect to $\Delta G^{*}$ are defined by $l^{*}, m^{*}, n^{*}$. When we set $m$ the length along a direction normal to epitaxial plane of NA, $m^{*}$ is the critical value in relation to the dimension of critical nucleus, which is given by

$$
m^{*}=\frac{2 \Delta \sigma}{\Delta g} .
$$

When $\mathrm{m}^{*}>1$, i.e., $\Delta g<2 \Delta \sigma$, three dimensional (3D) nucleation takes place. In the case of $\mathrm{m}^{*}<1$, i.e., $\Delta g>$ $2 \Delta \sigma$, two dimensional (2D) nucleation occurs. $\Delta G^{*}$ s of $3 \mathrm{D}$ and 2D nucleation $\left(\Delta G^{*}{ }_{3 \mathrm{D}}\right.$ and $\Delta G^{*}{ }_{2 \mathrm{D}}$, respectively) are expressed by

$$
\Delta G_{3 \mathrm{D}}^{*}(\Delta \sigma)=\frac{16 \sigma \sigma_{e} \Delta \sigma}{\Delta g^{2}} \text { for } m^{*}>1, \Delta g<2 \Delta \sigma
$$

and

\footnotetext{
${ }^{1}$ ADEKA Corporation, 5-2-13, Shirahata, Minami-ku, Saitama 336-0022, Japan

${ }^{2}$ Graduate School of Integrated Arts and Sciences, Hiroshima University, 1-7-1 Kagamiyama, Higashihiroshima 739-8521, Japan

*To whom correspondence should be addressed (Tel: +81-48-838-2244, Fax: +81-48-838-2250, E-mail: urushi@adeka.co.jp).
} 


$$
\Delta G_{2 \mathrm{D}}^{*}(\Delta \sigma)=\frac{4 \sigma \sigma_{e}}{\Delta g-\Delta \sigma} \quad \text { for } \quad m \leq 1, \Delta g \geq 2 \Delta \sigma,
$$

respectively. From eq 5 and thermodynamic relation given by

$$
\Delta g=\frac{\Delta h \Delta T}{T_{m}^{0}}
$$

where $\Delta h$ is enthalpy of fusion, $\Delta T$ is degree of supercooling and $T_{\mathrm{m}}{ }^{0}$ is equilibrium melting temperature, ${ }^{5}$ the supercooling temperature of conversion from $3 \mathrm{D}$ to $2 \mathrm{D}$ nucleation $\Delta T_{2 \mathrm{D} / 3 \mathrm{D}}$ is given by

$$
\Delta T_{2 \mathrm{D} / 3 \mathrm{D}}=\frac{2 \Delta \sigma T_{\mathrm{m}}^{0}}{\Delta h}
$$

\section{Importance of $\boldsymbol{\Delta} \boldsymbol{\sigma}$ for Study of NA}

Even CNT showed that $\Delta G^{*}$ depends on $\Delta \sigma$ as described in eqs 6 and 7, kinetic studies of nucleation based on CNT in the past have not paid much attention to $\Delta \sigma$ and $\Delta T_{2 \mathrm{D} / 3 \mathrm{D}}$. As some heterogeneity must inherently be included without trying to remove it carefully, we have to take heterogeneous effect, i.e., $\Delta \sigma$, into account. NA has much smaller $\Delta \sigma$ than a mere heterogeneity so that $\Delta T_{2 \mathrm{D} / 3 \mathrm{D}}$ of a polymer with NA is smaller than that without NA. Therefore we have to choose the appropriate formulation of $3 \mathrm{D}$ or $2 \mathrm{D}$ nucleation as to $\Delta \sigma$ and $\Delta T_{2 \mathrm{D} / 3 \mathrm{D}}$. But the evaluation method of $\Delta \sigma$ has not been established yet. In this study we will show how to determine $\Delta \sigma$.

When we assume that $\sigma_{0 \mathrm{~s}}$ changes from 0 to $\sigma, \Delta \sigma$ changes from 0 to $\sigma$, i.e.,

$$
\Delta \sigma=0 \sim \sigma \quad \text { or } \quad \Delta \sigma / \sigma=0 \sim 1, \text { for } \quad \sigma_{0 \mathrm{~s}}=0 \sim \sigma .
$$

In this study we use $\Delta \sigma / \sigma$. When $\Delta \sigma / \sigma=0$, the nucleus becomes an ideal NA. This corresponds to the secondary nucleation. When $\Delta \sigma / \sigma=1$, the epitaxy between heterogeneity and nucleus is negligible. $\Delta G^{*}$ decreases with decrease of $\Delta \sigma / \sigma$. This results in acceleration of $I$. In the case of heterogeneity with small $\Delta \sigma / \sigma$, i.e., $\Delta \sigma / \sigma \ll 1$, it is named NA. As $\Delta \sigma / \sigma$ of NA is not commonly given, we will name the heterogeneity as NA when

$$
\Delta \sigma / \sigma<0.3
$$

is satisfied in this study.

\section{Epitaxy of NAs}

Epitaxy comes from an interaction between two contact planes of the crystals. Thus efficiency of epitaxy should be controlled by two dimensional lattice matches in the contact planes. Degree of misfit $(\delta)$ for an azimuthal orientation in the contact planes is defined by

$$
\delta=\frac{d-d_{s}}{d} \times 100(\%)
$$

where $d$ and $d_{\mathrm{s}}$ are lengths of the approximate least common multiple of lattice constants of an overgrowth layer and substrate, respectively. ${ }^{6}$ Epitaxy should take place when absolute value of $\delta$ is small. (a)

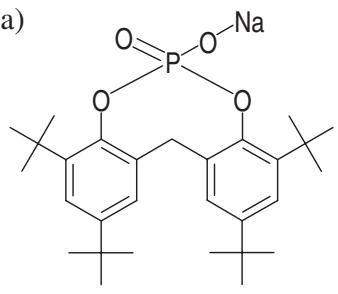

(b)

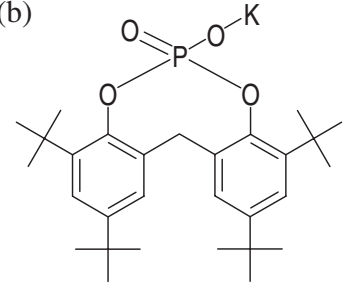

Figure 1. Chemical structure of (a) NA-11 and (b) NA-11K.

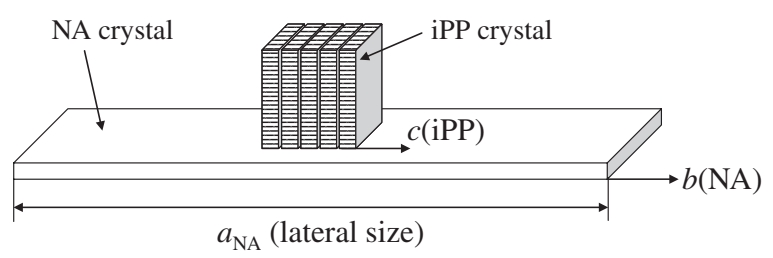

Figure 2. Epitaxial relationship between NA-11 and iPP crystals.

In this study, iPP was used as a typical polymer. As a typical high-performance NA for iPP, sodium 2,2'-methylene-bis-(4,6di-t-butylphenylene)phosphate (ADEKA Corp., NA-11) was used. The structure of NA-11 is shown in Figure 1a. Kawaguchi et al. reported that the epitaxial relationship between NA-11 and iPP crystals, ${ }^{7}$

$$
b(\mathrm{NA}-11) / / c(\mathrm{iPP})
$$

where $b$ (NA-11) and $c$ (iPP) are crystal lattice constants of $b$ of NA-11 and $c$ of iPP, respectively. This is schematically shown in Figure 2. As the other azimuthal orientation in the contact planes is not specified, we will only evaluate lattice matching for one azimuthal orientation in eq 13. In this study, substrate, i.e., NA, will be changed for a polymer, i.e., iPP. Eq 12 can be rewritten as

$$
\delta^{\prime}=\frac{d(\mathrm{iPP})-d(\mathrm{NA})}{d(\mathrm{iPP})} \times 100(\%)
$$

where $\delta^{\prime}$ is degree of misfit between NA and iPP crystals, $d(\mathrm{iPP})$ and $d(\mathrm{NA})$ are the lattice constants for an azimuthal orientation in the contact planes. As $b$ (NA-11) and $c$ (iPP) are

$$
b(\mathrm{NA}-11)=0.608(\mathrm{~nm}) \quad \text { and } \quad c(\mathrm{iPP})=0.65(\mathrm{~nm}),
$$

respectively, ${ }^{5,8} \delta^{\prime}$ given by eq 14 becomes

$$
\delta^{\prime}=+6.5(\%) \text {. }
$$

Crystal lattice match between NA-11 and iPP is not good enough. In order to decrease $\delta^{\prime}$, the sodium $\mathrm{Na}$ atom in NA-11 was substituted by a larger atom of potassium K, i.e., potassium 2,2'-methylene-bis-(4,6-di-t-butylphenylene) phosphate (Figure $1 \mathrm{~b}$ ) was synthesized. As a typical conventional NA, sodium benzoate was used. The unit cell structure of sodium benzoate has not been reported. As sodium benzoate shows lower performance, $\Delta \sigma$ of sodium benzoate should be larger than that of NA-11. 


\section{Purpose}

The first purpose of this study is to clarify the free energy of forming critical nucleus $\Delta G^{*}(\Delta \sigma)$ dependence of the nucleation rate $I$ based on the nucleation theory, where $\Delta \sigma$ is a kinetic parameter reflected in efficiency of epitaxy. Clarification of the effect of $\Delta G^{*}(\Delta \sigma)$ on $I$ is important for understanding a role of epitaxy of NA in heterogeneous nucleation. The second purpose of this study is to clarify the effect of $\Delta \sigma$ on the geometrical manner of nucleation, i.e., three or two dimensional (3D or 2D) ones, and to establish the evaluation method of $\Delta \sigma$. Understanding of supercooling temperature of $3 \mathrm{D}-2 \mathrm{D}$ conversion $\Delta T_{2 \mathrm{D} / 3 \mathrm{D}}$ is the need for determination of $\Delta \sigma$. And the evaluation method of $\Delta \sigma$ should be useful for quantitative analysis of the ability of NA.

\section{THEORETICAL}

\section{Conversion of $I(\Delta \sigma)$ at $\Delta T_{2 \mathrm{D} / 3 \mathrm{D}}$}

Formulation of $I(\Delta \sigma)$ as a function of $\Delta T$ is useful for kinetic study. Insertion of eqs 6 or 7 into eq 1 gives $I$ of $3 \mathrm{D}$ or $2 \mathrm{D}$ nuclei ( $I_{3 \mathrm{D}}$ or $I_{2 \mathrm{D}}$, respectively). Here $I_{3 \mathrm{D}}$ and $I_{2 \mathrm{D}}$ are divided by $I_{0}$ to eliminate the effect of $I_{0}$ on $I . I_{3 \mathrm{D}} / I_{0}$ and $I_{2 \mathrm{D}} / I_{0}$ are given by

$$
\begin{array}{r}
\frac{I_{3 \mathrm{D}}(\Delta \sigma)}{I_{0}}=\exp \left(-\frac{\Delta G_{3 \mathrm{D}}^{*}}{k T_{\mathrm{c}}}\right)= \\
\text { for } \Delta g<2 \Delta \sigma
\end{array}
$$

where

$$
\mathrm{C}(\Delta \sigma)=\frac{16 \sigma \sigma_{\mathrm{e}} \Delta \sigma T_{\mathrm{m}}^{0^{2}}}{k \Delta h^{2}}
$$

and

$$
\begin{array}{r}
\frac{I_{2 \mathrm{D}}(\Delta \sigma)}{I_{0}}=\exp \left(-\frac{\Delta G_{2 \mathrm{D}}^{*}}{k T_{\mathrm{c}}}\right)=\exp \left\{-\frac{\alpha(\Delta \sigma) \mathrm{B}}{T_{\mathrm{c}} \Delta T}\right\} \\
\text { for } \Delta g \geq 2 \Delta \sigma
\end{array}
$$

where

$$
\begin{aligned}
& \alpha(\Delta \sigma) \equiv \frac{\Delta g}{\Delta g-\Delta \sigma}=\frac{\Delta h \Delta T}{\Delta h \Delta T-\Delta \sigma T_{\mathrm{m}}^{0}} \\
& \text { and } \quad \mathrm{B}=\frac{4 \sigma \sigma_{\mathrm{e}} T_{\mathrm{m}}^{0}}{k \Delta h},
\end{aligned}
$$

respectively.

When $\Delta \sigma / \sigma=0, \alpha$ in eq 20 becomes 1 . Therefore we can rewrite eq 19 as

$$
\begin{aligned}
\frac{I_{2 \mathrm{D}}(\Delta \sigma=0)}{I_{0}} & =\exp \left(-\frac{\Delta G_{2 \mathrm{D}}^{*}(\Delta \sigma=0)}{k T_{\mathrm{c}}}\right) \\
& =\exp \left\{-\frac{\mathrm{B}}{T_{\mathrm{c}} \Delta T}\right\} .
\end{aligned}
$$

From the eqs 21 and A.3,

$$
\frac{I_{2 \mathrm{D}}(\Delta \sigma=0)}{I_{0}}=\left(\frac{V}{V_{0}}\right)^{\gamma}
$$

where $V$ and $V_{0}$ are the lateral growth rate and a prefactor, respectively, $\gamma=1$ or 3 corresponds to single or multi nucleation, respectively. (a) $T_{\mathrm{c}} /{ }^{\circ} \mathrm{C}$

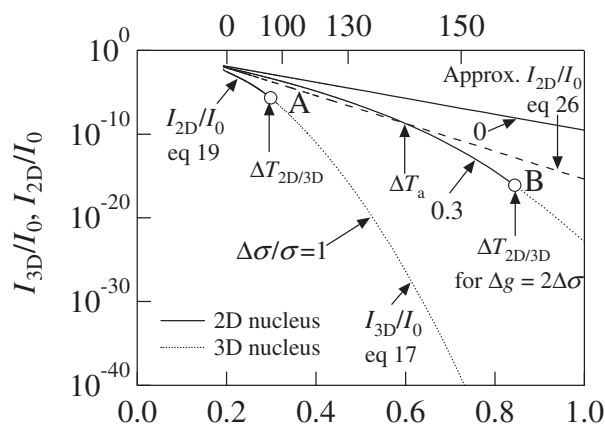

(b)

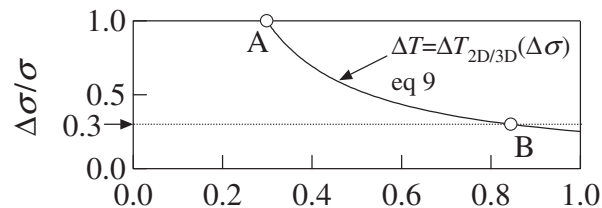

(c)

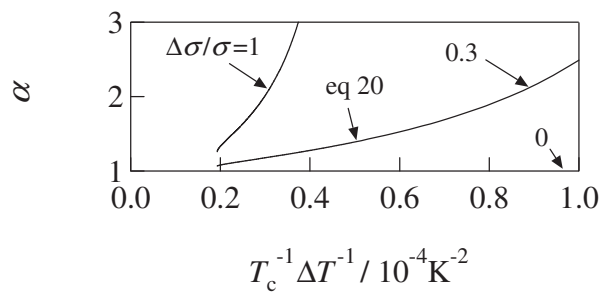

Figure 3. (a) Theoretical curves of $I_{2 \mathrm{D}} / \mathrm{l}_{0}$ and $I_{3 \mathrm{D}} / \mathrm{l}_{0}$ of iPP against $T_{\mathrm{c}}^{-1} \Delta T^{-1}$ as a parameter of $\Delta \sigma / \sigma$. Dimension of the shape of primary nucleus changes at $\Delta T_{2 \mathrm{D} / 3 \mathrm{D}}$ where $\Delta g=2 \Delta \sigma$. Upper axis indicates $T_{\mathrm{c}}$. Solid line and curves show $I_{20} / I_{0}$ and dotted curves show $I_{3 \mathrm{D}} / I_{0}$. I decreases with increase of $\Delta \sigma / \sigma$ for a constant $T_{\mathrm{c}}$. A and B corresponds to those in (b). Broken line shows approximated $I_{20} / I_{0}$ of eq 26 in the range of $\Delta T=30-50 \mathrm{~K}$. (b) Theoretical curve of $\Delta \sigma / \sigma$ against $T^{-1} \Delta T^{-1} . \Delta T_{2 \mathrm{D} / 3 \mathrm{D}}$ decreases with increase of $\Delta \sigma / \sigma$. (c) Theoretical curves of $\alpha$ against $T_{c}^{-1} \Delta T^{-1}$ as a parameter of $\Delta \sigma / \sigma . \alpha$ increases with increase of $\Delta \sigma / \sigma . \alpha$ approaches to 1 with decrease of $\Delta \sigma / \sigma$.

\section{Approximated Analysis of $I_{2 \mathrm{D}}$ for $\boldsymbol{\alpha} \cong$ constant}

In $\Delta G^{*}{ }_{2 \mathrm{D}}=k \alpha \mathrm{B} / \Delta T$ in eq $19, \alpha$ given by eq 20 is a function of $\Delta \sigma$ and $\Delta T$, i.e.,

$$
\alpha=\alpha(\Delta \sigma, \Delta T) .
$$

This is the reason why $I_{2 \mathrm{D}} / I_{0}$ of eq 19 shows curvature against $T_{\mathrm{c}}^{-1} \Delta T^{-1}$ in Figure 3. When $\Delta \sigma / \sigma$ is small and observed range of $\Delta T$ is limited to rather narrow range, $\alpha$ can be regarded to be constant as shown in Figure 3c. In this case $\alpha$ is approximated by a constant $\alpha_{\mathrm{a}}$ which is given by

$$
\alpha_{\mathrm{a}} \equiv \alpha\left(\Delta \sigma, \Delta T_{\mathrm{a}}\right)
$$

where $\Delta T_{\mathrm{a}}$ is averaged $\Delta T$ between the smallest and largest $\Delta T\left(\Delta T_{\mathrm{S}}\right.$ and $\Delta T_{\mathrm{L}}$, respectively). $\Delta T_{\mathrm{a}}$ is defined by

$$
\frac{1}{\Delta T_{\mathrm{a}}}=\frac{\frac{1}{\Delta T_{\mathrm{S}}}+\frac{1}{\Delta T_{\mathrm{L}}}}{2} .
$$

In this case $\alpha \mathrm{B}$ becomes constant. Therefore $I_{2 \mathrm{D}} / I_{0}$ against $T_{\mathrm{c}}^{-1} \Delta T^{-1}$ in Figure 3 can be approximated by a straight line given by 
Table I. Parameters of iPP, $\sigma, \sigma_{\mathrm{e}}, \Delta h$ and $T_{\mathrm{m}}{ }^{0}$

\begin{tabular}{cc}
\hline$\sigma / \mathrm{Jm}^{-2}$ & $1.15 \times 10^{-29}$ \\
$\sigma_{\mathrm{e}} / \mathrm{Jm}^{-2}$ & $5.5 \times 10^{-210}$ \\
$\sigma \sigma_{\mathrm{e}} / \mathrm{J}^{2} \mathrm{~m}^{-4}$ & $6.33 \times 10^{-49,10}$ \\
$\Delta h / \mathrm{Jm}^{-3}$ & $2.09 \times 10^{811}$ \\
$T_{\mathrm{m}}^{0} / \mathrm{K}$ & $455.75^{12-14}$ \\
\hline
\end{tabular}

$$
\frac{I_{2 \mathrm{D}}}{I_{0}}=\exp \left(-\frac{\alpha_{\mathrm{a}} \mathrm{B}}{T_{\mathrm{c}} \Delta T}\right)
$$

Broken line in Figure $3 \mathrm{a}$ is approximated $I_{2 \mathrm{D}} / I_{0}$ of eq 26 in the range of $\Delta T=30-50 \mathrm{~K}$. The broken line was shifted to intersect with the curve of $I_{2 \mathrm{D}} / I_{0}$ at $\Delta T_{\mathrm{a}}$ by changing $I_{0}$. This approximation will be confirmed in Figure 11.

\section{EXPERIMENTAL}

\section{Materials and Sample Preparation}

Typical polymer of iPP (the number average molecular weight $M_{\mathrm{n}}=6.4 \times 10^{4}$, the weight average molecular weight $M_{\mathrm{w}}=3.0 \times 10^{5}$, the molecular weight distribution $M_{\mathrm{w}} / M_{\mathrm{n}}=$ 4.6 and a meso pentad fraction $[\mathrm{mmmm}]=97 \%$ ) was used. Parameters of iPP crystals used in this study are shown in Table I. ${ }^{9-14} T_{\mathrm{m}}{ }^{0}$ of iPP was estimated from the relationship between $M_{\mathrm{n}}$ and tacticity. ${ }^{12-14}$ Repeating unit of iPP was assumed that the folding direction is along (110).

Typical high-performance NA of sodium 2,2'-methylenebis-(4,6-di-t-butylphenylene)phosphate (ADEKA Corp., NA11) was used. To improve lattice matching between NA-11 and iPP crystals, potassium 2,2'-methylene-bis-(4,6-di-t-butylphenylene)phosphate (NA-11K) shown in Figure 1b was synthesized as follows. NA-11 of $25.4 \mathrm{~g} \quad(0.05 \mathrm{~mol})$, methanol (KOKUSAN chemical Co., Ltd.) of $250 \mathrm{~mL}$ and distilled water of $50 \mathrm{~mL}$ were mixed, and then $1 \mathrm{~N} \mathrm{HCl}$ aqueous solution (KOKUSAN chemical Co., Ltd.) of $60 \mathrm{~mL}(0.06 \mathrm{~mol})$ was dropped onto the mixture at room temperature $T_{\mathrm{R}}$ with vigorous stirring. After finished dropping, the mixture was stirred at $T_{\mathrm{R}}$ for $1 \mathrm{~h}$. The solid mass which precipitated was collected by filtration and washed three times by water of each $50 \mathrm{~mL}$. Obtained crude product was solved in methanol of $600 \mathrm{~mL}$, heated up to $60^{\circ} \mathrm{C}$. Distilled water of $600 \mathrm{~mL}$ was dropped onto the solution at $60^{\circ} \mathrm{C}$, and then the mixture was cooled to $T_{\mathrm{R}}$. Precipitated product was filtered and dried. The yield of the product was $20.9 \mathrm{~g}(86.1 \%)$. The product of $12.2 \mathrm{~g}$ $(0.025 \mathrm{~mol})$ and methanol of $120 \mathrm{~mL}$ was mixed, and subsequently $1 \mathrm{~N} \mathrm{KOH}$ aqueous solution (KOKUSAN chemical Co., Ltd.) of $25.8 \mathrm{~mL}(0.0258 \mathrm{~mol})$ was added onto the mixture little by little at $T_{\mathrm{R}}$ with vigorous stirring, followed by stirring at $T_{\mathrm{R}}$ for $1 \mathrm{~h}$. After that, about $60 \mathrm{~mL}$ of the solvent was evaporated under reduced pressure at $T_{\mathrm{R}}$. The product was filtered and washed three times by water of each $20 \mathrm{~mL}$, and then dried. The yield of the product (NA-11K) was $9.1 \mathrm{~g}$ $(69.5 \%)$. The product assignment and purity were analyzed by means of ${ }^{1} \mathrm{H}$ NMR (JEOL, Ltd., FT NMR Lambda 400), IR spectroscopy (SHIMADZU Co., FT/IR-7500) and ICP
(SHIMADZU, ICPS-7500). The purity was over $99 \%$ and sodium content was under the detective limit $(<1 \mathrm{ppm})$. The obtained NA-11K crystal was grinded by a vibrating mill (CMT Co., Ltd., TI-500ET). As a conventional NA, sodium benzoate (ADEKA PALMAROLE SAS, MI.NA.08) was used. Hereafter this material will be named "Na-Bz."

Mean lateral size of NAs $\left(\overline{a_{\mathrm{NA}}}\right)$ was derived from the size distribution $f\left(a_{\mathrm{NA}}\right)$ which is measured from the SEM photographs. ${ }^{2}$ NA crystal was uniformly mixed with solution of iPP and xylene at $130^{\circ} \mathrm{C}$. The suspension was quenched to $T_{\mathrm{R}}$, and then dried. The sample of iPP mixed with NA will be described as iPP/NA.

\section{Instrumentals}

OM and SEM. Morphology of NA crystal was observed by means of optical microscope (OM), OLYMPUS BX-51, and scanning electron microscope (SEM), Topcon SM-520.

WAXS. Wide angle X-ray scattering (WAXS) measurement was performed on the diffractometer, (Rigaku Corp., RINTUltimaIII). All the samples were examined by $\mathrm{CuK} \alpha$ radiation monochromatized by graphite crystal at $T_{\mathrm{R}}$. Divergence slit of $1 / 2^{\circ}$ was used. Slit width for the incidence and receiving beam were 0.73 and $0.3 \mathrm{~mm}$, respectively. Scattering intensity $I_{\mathrm{x}}(2 \theta)$ was obtained by means of scintillation counter.

DSC. DSC measurement was conducted by means of Diamond DSC (PerkinElmer Inc.). Temperature was calibrated by using melting temperature of standard materials, indium and tin. The sample weighed about $2 \mathrm{mg}$ was put in the aluminum pan and crimped with the aluminum rid. $C_{\mathrm{NA}}$ of all samples was $0.1 \mathrm{wt} \%$. The sample was heated up to $230^{\circ} \mathrm{C}$ at the cooling rate of $50 \mathrm{~K} / \mathrm{min}$ and then held for $5 \mathrm{~min}$. After that the sample was cooled to $50^{\circ} \mathrm{C}$ at the rate of $10 \mathrm{~K} / \mathrm{min}$. Crystallization peak temperature $\left(T_{\mathrm{c}}\right)$ was observed. Baseline was leveled by using the software (PerkinElmer Inc., Pyris).

\section{Crystallization of iPP}

Crystallization behaviors of iPP/NA were observed by using OM, OLYMPUS BX-51, with hot stage, LINKAM LK-600PM, under nitrogen flow of $50 \mathrm{~mL} / \mathrm{min}$. Temperature of the hot stage was calibrated by using melting temperature of standard materials, indium and tin. Sample was once melted at $210^{\circ} \mathrm{C}$ for $1 \mathrm{~min}$, and then crystallized in the range of $T_{\mathrm{c}}=$ $127.0-148.8^{\circ} \mathrm{C}$. The time $t$ dependence of the number density $v(t)$ of iPP crystals which are larger than $2 \mu \mathrm{m}$ in lateral size was observed. $I$ is defined by

$$
I \equiv \frac{d \nu(t)}{d t}
$$

\section{RESULTS AND DISCUSSION}

\section{Theoretical Prediction of Significant Role of $\Delta \sigma$ in $I(\Delta T)$}

It is interesting to predict how $\Delta \sigma$ affects $I(\Delta T)$ significantly when $\Delta \sigma$ decreases from 1 to 0 , because $\Delta \sigma$ represents the ability of NA. And the effect of $\Delta \sigma$ on $I(\Delta T)$ has little studied. $I / I_{0}$ vs. $T_{\mathrm{c}}^{-1} \Delta T^{-1}$ can be simulated as a parameter of 
(a)

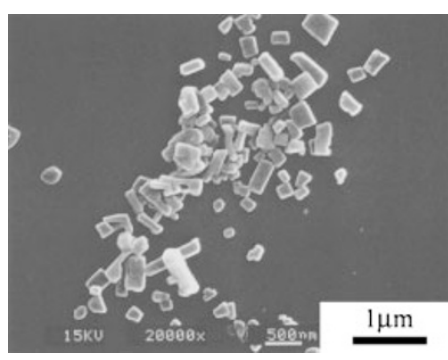

(b)

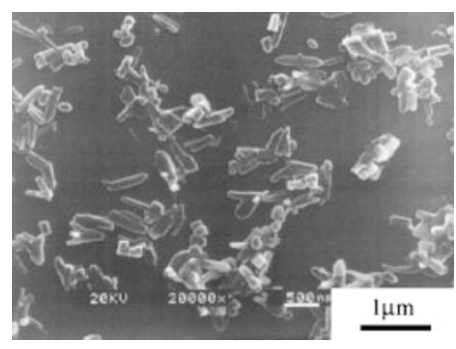

(c)

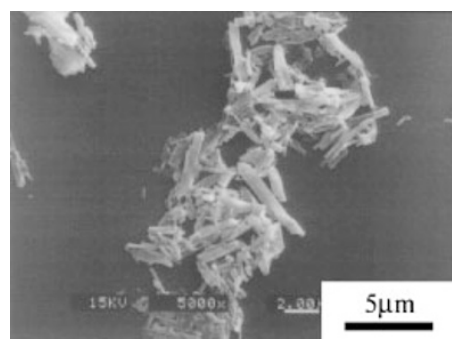

(d)

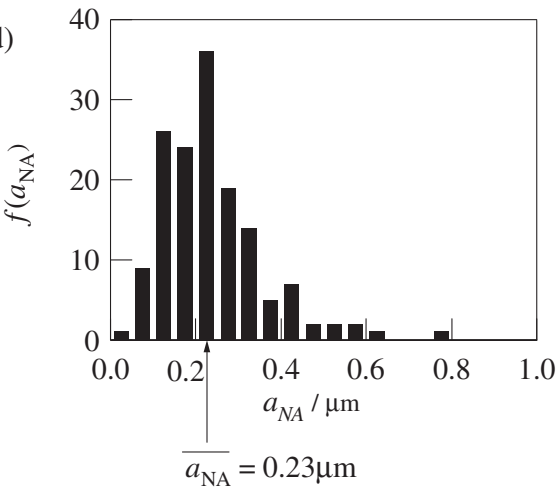

(e)

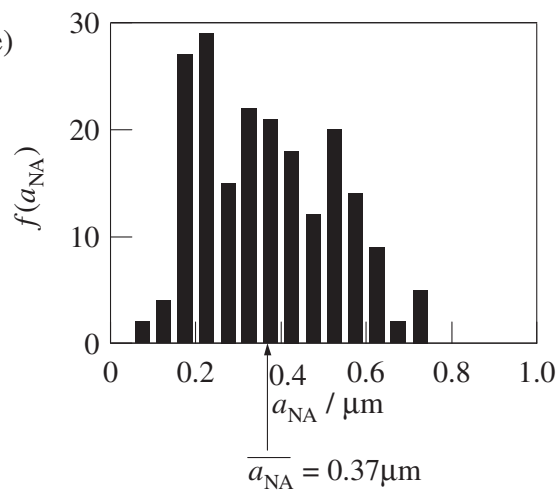

(f)

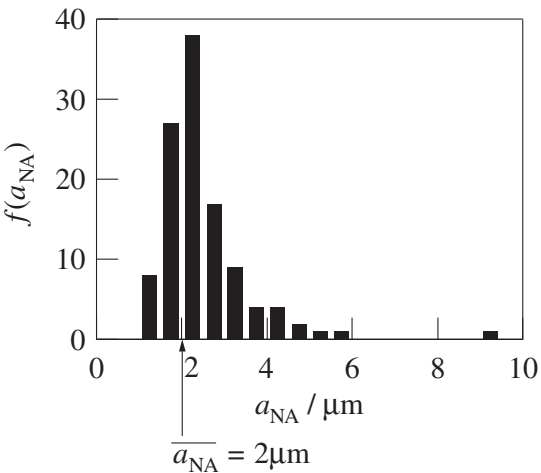

Figure 4. Morphology and $f\left(a_{N A}\right)$ of NA crystals. Scanning electron micrographs of (a) NA-11, (b) NA-11K and (c) Na-Bz crystals, and $f\left(a_{N A}\right)$ and $\overline{a_{N A}}$ of (d) NA11, (e) NA-11K and (f) Na-Bz crystals.

$\Delta \sigma / \sigma$ by using eqs 17,19 and $\sigma \sigma_{e}$ from literature. Parameters of iPP are shown in Table I. ${ }^{9-14}$ Theoretical curves of $I / I_{0}$ against $T_{\mathrm{c}}^{-1} \Delta T^{-1}$ as a parameter of $\Delta \sigma / \sigma$ are shown in Figure 3a. Solid and dotted curves show $I_{2 \mathrm{D}} / I_{0}$ and $I_{3 \mathrm{D}} / I_{0}$, respectively. Nucleus changes from $2 \mathrm{D}$ to $3 \mathrm{D}$ with decrease of $\Delta T$ (i.e., increase of $T_{\mathrm{c}}$ ). The conversion from $2 \mathrm{D}$ to $3 \mathrm{D}$ nucleus is seen at $\Delta T=\Delta T_{2 \mathrm{D} / 3 \mathrm{D}}$ given by eq 9 which corresponds to $\Delta g=2 \Delta \sigma . \Delta T_{2 \mathrm{D} / 3 \mathrm{D}}$ decreased with decrease of $\Delta \sigma / \sigma$ as shown in Figure $3 \mathrm{~b}$. $\mathrm{A}$ and $\mathrm{B}$ in Figure $3 \mathrm{a}$ corresponds to those in Figure $3 \mathrm{~b}$. Figure $3 \mathrm{c}$ shows the curves of $\alpha$ in eq 20 against $T_{\mathrm{c}}^{-1} \Delta T^{-1}$ as a parameter of $\Delta \sigma / \sigma . \alpha$ takes the value which is slightly larger than 1. $\alpha$ increases with increasing $T_{\mathrm{c}}^{-1} \Delta T^{-1}$. With decreasing $\Delta \sigma / \sigma, \alpha$ approaches to 1. $I / I_{0}$ significantly increase with decrease of $\Delta \sigma / \sigma$. For example, $I / I_{0}$ increased by 38 orders of magnitude for $\Delta T=30 \mathrm{~K}$ and 12 orders of magnitude for $\Delta T=50 \mathrm{~K}$ with decreasing $\Delta \sigma / \sigma$ from 1 to 0 . In the case of NA where $\Delta \sigma / \sigma<0.3, I$ increases by $10^{6}$ times for $\Delta T=30 \mathrm{~K}$ and 60 times for $\Delta T=50 \mathrm{~K}$ with decrease of $\Delta \sigma / \sigma$ from 0.3 to $0 . I$ significantly depends on $\Delta \sigma / \sigma$. Therefore $\Delta \sigma / \sigma$ is essentially important for acceleration of nucleation.

\section{Morphology and Size of NA Crystals}

Morphology of NA-11, NA-11K and Na-Bz crystals were observed by means of SEM as shown in Figure 4a, 4b and 4c, respectively. All NA crystals showed rectangular plate shape. As will be shown in Figure 7, NA-11 and NA-11K crystals have the same shape as their large single crystals. The crystals of NAs have a tendency to cleave along the lateral direction. This results in the same shape of grinded crystal as their large single crystal and preservation of epitaxial lattice plane. Size distributions $\left(f\left(a_{\mathrm{NA}}\right)\right)$ of NA-11, NA-11K and Na-Bz crystals are 

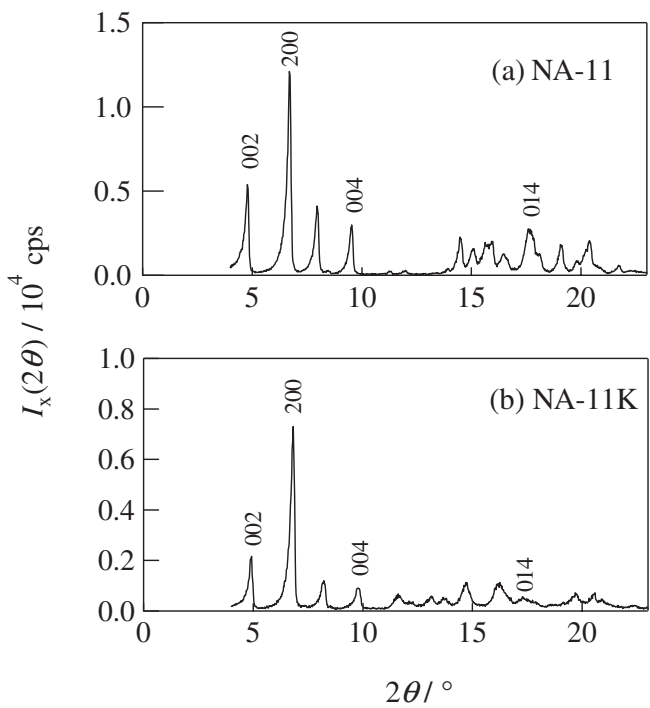

Figure 5. WAXS diffraction patterns of (a) NA-11 and (b) NA-11K crystals at $T_{R}$.

shown in Figure $4 \mathrm{~d}, 4 \mathrm{e}$ and $4 \mathrm{f}$, respectively. $f\left(a_{\mathrm{NA}}\right)$ s of all NA crystals were broad because they were prepared by "top down" method of mechanical grinding as shown in the previous paper. ${ }^{2}$ $\overline{a_{N A}}$ of NA-11, NA-11K and Na-Bz crystals were $0.23 \mu \mathrm{m}$, $0.37 \mu \mathrm{m}$ and $2.0 \mu \mathrm{m}$, respectively. $\overline{a_{N A}}$ of Na-Bz crystals was much larger than that of NA-11 and NA-11K crystals. As $I \propto$ ${\overline{a_{N A}}}^{-1}$ was confirmed in the previous study, $I$ of $\mathrm{iPP} / \mathrm{Na}-\mathrm{Bz}$ is expected to be smaller than $I$ of iPP/NA-11 or iPP/NA-11K.

\section{Unit Cell Structure of iPP and NA Crystals}

Unit cell structure of iPPs mixed with NAs belongs to $\alpha$ form as shown for iPP/NA-11 in the previous paper. ${ }^{2}$ Unit cell structure of NA-11K crystal was determined as the same $\alpha$ form as that of NA-11 crystal, ${ }^{5}$ because the WAXS patterns of them were the same in the range of $2 \theta=4 \sim 10^{\circ}$ as shown in Figure 5. Crystal lattice constant of $b$ of NA-11K ( $b(\mathrm{NA}-11 \mathrm{~K}))$ was $0.634 \mathrm{~nm}$. As expected in introduction, $b(\mathrm{NA}-11 \mathrm{~K})$ was larger than $b$ (NA-11). $\delta^{\prime}$ of NA-11K was $+2.5 \%$, which is smaller than that of NA-11 $\left(\delta^{\prime}(\mathrm{NA}-11)=+6.5 \%\right)$. Thus $\Delta \sigma / \sigma$ of NA-11K is expected to be smaller than that of NA-11.

\section{Effect of NAs on Crystallization Observed by Means of DSC}

Crystallization behaviors of iPP/NA-11, iPP/NA-11K, iPP/ $\mathrm{Na}-\mathrm{Bz}$ and iPP observed by means of DSC are shown in Figure $6 . C_{\mathrm{NA}}$ was $0.1 \mathrm{wt} \%$. As iPP includes some heterogeneity unintentionally, hereafter we name it iPP/het. Crystallization peak temperature $T_{\mathrm{c}, \mathrm{p}}$ of iPP/NA-11, iPP/NA-11K, $\mathrm{iPP} / \mathrm{Na}-\mathrm{Bz}$ and $\mathrm{iPP} /$ het were $132.3,131.8,122.0$ and $117.6^{\circ} \mathrm{C}$, respectively. $T_{c, p}$ of $\mathrm{iPP} / \mathrm{NA}-11$ and $\mathrm{iPP} / \mathrm{NA}-11 \mathrm{~K}$ is about $15^{\circ} \mathrm{C}$ higher than that of iPP/het. $T_{c, p}$ of iPP/Na-Bz is only $4.4{ }^{\circ} \mathrm{C}$ higher than that of $\mathrm{iPP} /$ het. This indicates that the ability of $\mathrm{Na}-\mathrm{Bz}$ as NA is lower than that of NA-11 or NA-11K as will be shown in detail later.

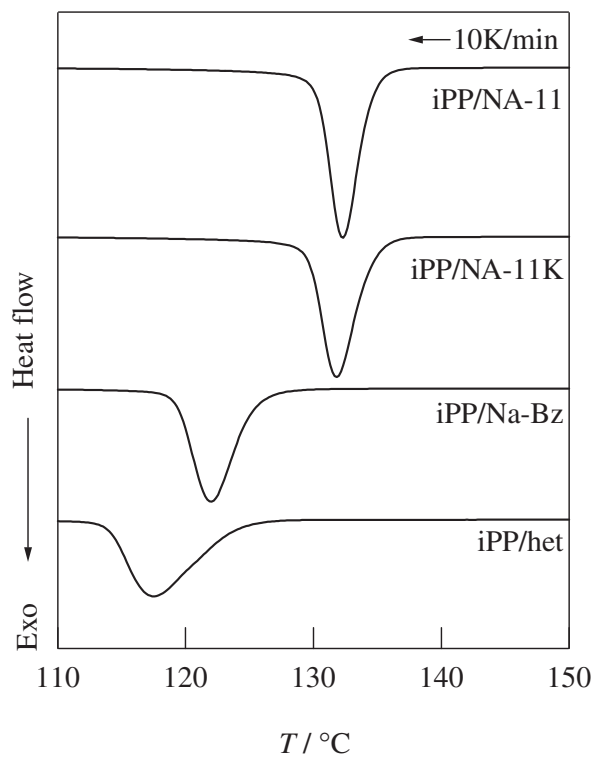

Figure 6. Crystallization behavior of iPP/NAs measured by means of DSC Cooling rate was $10 \mathrm{~K} / \mathrm{min}$. $C_{\mathrm{NA} / \mathrm{PPP}}=0.1 \mathrm{wt} \%$.

(a)
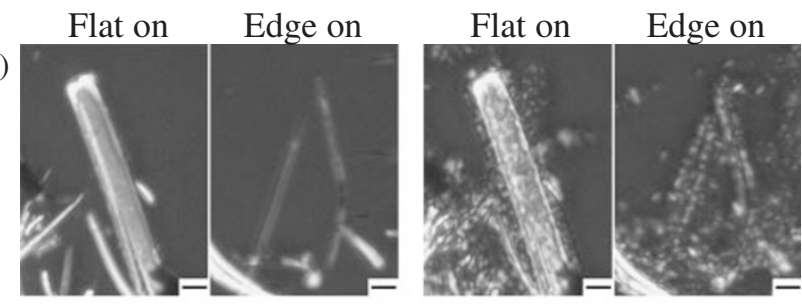

(b)

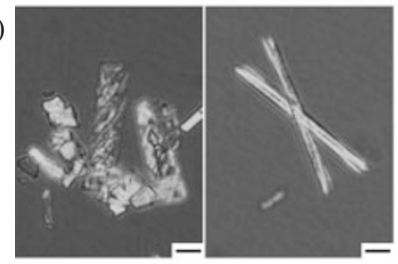

(c)

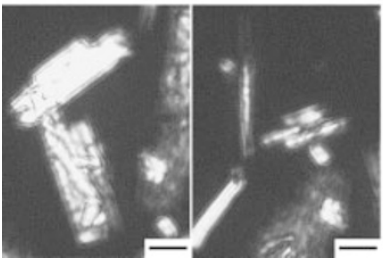

$\mathrm{t}=0 \mathrm{~min}$
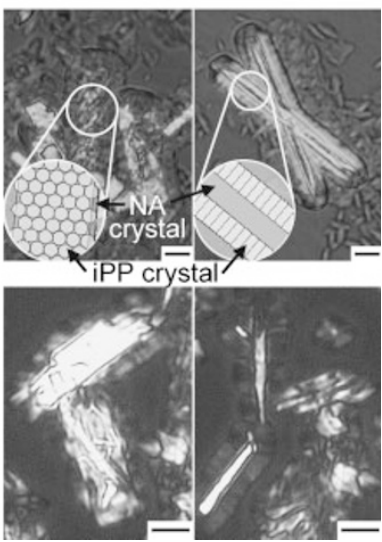

$\mathrm{t}=5 \mathrm{~min}$

Figure 7. Isothermal crystallization of iPPs mixed with large crystals of (a) NA-11, (b) NA-11K and (c) Na-Bz at $T_{\mathrm{c}}=138.8^{\circ} \mathrm{C}$. Scale bar is $10 \mu \mathrm{m}$.

\section{Optical Evidence of Epitaxial Growth of iPP on Large Single Crystals of NA}

Epitaxial growth of iPP on large single crystals of NA-11, NA-11K and Na-Bz observed at $T_{\mathrm{c}}=138.8^{\circ} \mathrm{C}$ were shown in Figure $7 \mathrm{a}, 7 \mathrm{~b}$ and $7 \mathrm{c}$, respectively. Schematic illustrations of morphology as typical examples are shown in the photograph of Figure $7 \mathrm{~b} \quad(\mathrm{t}=5 \mathrm{~min})$. The iPP lamella crystals grew epitaxially perpendicular to the largest plane of NA crystals. 


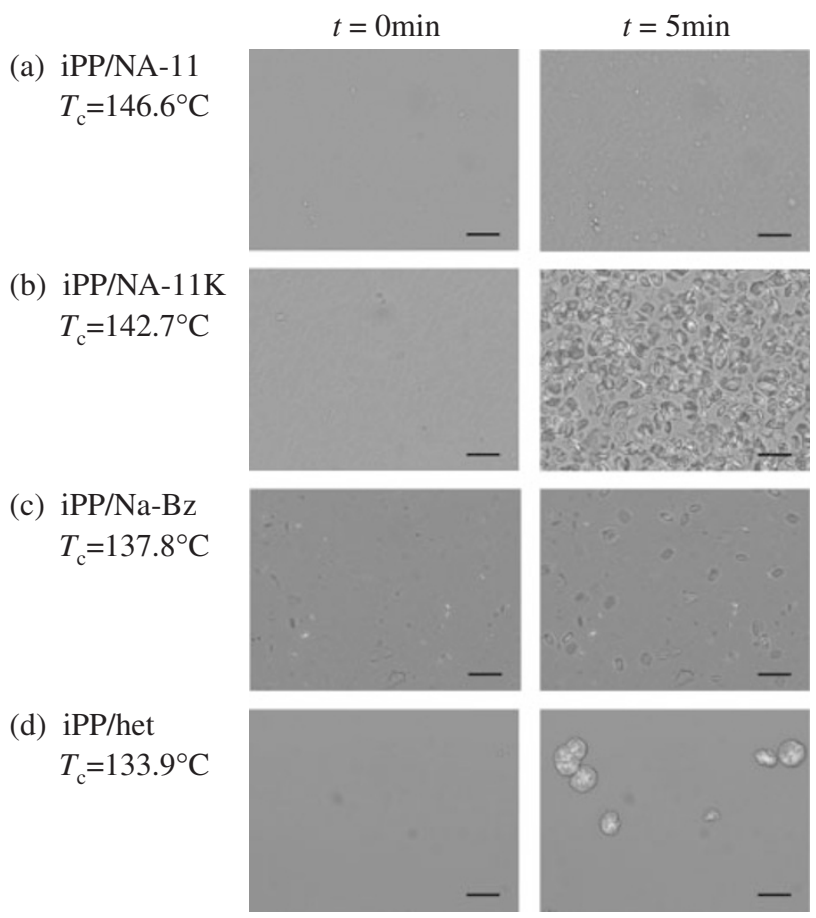

Figure 8. Isothermal crystallization behavior of (a) iPP/NA-11, (b) iPP/NA$11 \mathrm{~K}$, (c) iPP/Na-Bz and (d) iPP/het. $C_{\mathrm{NA} / \mathrm{iPP}}$ and $\overline{\mathrm{a}_{\mathrm{NA}}}$ are shown in Table II. Scale bar is $50 \mu \mathrm{m}$.

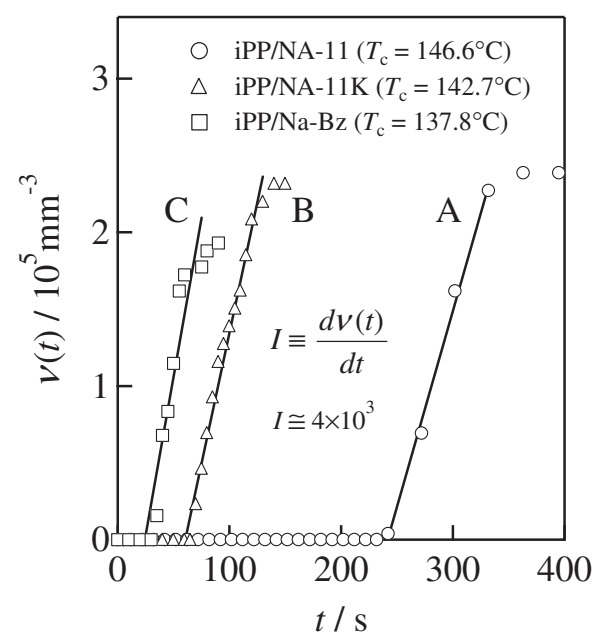

Figure 9. Plots of $v(t)$ of iPP/NA-11, iPP/NA-11K and iPP/Na-Bz against $t$. Nearly the same $I$ was observed at different $T_{\mathrm{c}}$. A, B and $\mathrm{C}$ corresponds to those in Figure 10.

The lamella crystals showed "transcrystal" like morphology. The density of iPP nucleus on the surface of Na-Bz crystal is less than that on the surface of NA-11 and NA-11K crystals. This indicates that efficiency of epitaxy of $\mathrm{Na}-\mathrm{Bz}$ is lower than that of NA-11 and NA-11K.

Isothermal crystallization behavior of iPP/NA-11, iPP/NA$11 \mathrm{~K}, \mathrm{iPP} / \mathrm{Na}-\mathrm{Bz}$ and $\mathrm{iPP} /$ het are shown in Figure $8 \mathrm{a}, 8 \mathrm{~b}, 8 \mathrm{c}$ and $8 \mathrm{~d}$, respectively. $T_{\mathrm{c}} \mathrm{s}$ of iPP/NA-11, iPP/NA-11K and iPP/ $\mathrm{Na}-\mathrm{Bz}$ correspond to those in Figure 9, respectively. $C_{\mathrm{NA}}$ and $\overline{a_{N A}}$ are shown in Table II. A larger number of iPP crystals
Table II. $\overline{\mathrm{a}_{\mathrm{NA}}}$ of various NAs and $C_{\mathrm{NA}}, \Delta \sigma / \sigma$ and $l_{0}$ of $\mathrm{iPP} / \mathrm{NA}-11$, $\mathrm{iPP} / \mathrm{NA}-11 \mathrm{~K}, \mathrm{iPP} / \mathrm{Na}-\mathrm{Bz}$ and $\mathrm{iPP} /$ het. $\Delta \sigma / \sigma$ and $l_{0}$ were obtained from the fit of eq 19 to the experimental plots

\begin{tabular}{ccccc}
\hline NA & $\overline{\mathrm{a}_{\mathrm{NA}}}(\mu \mathrm{m})$ & $C_{\mathrm{NA}}(\mathrm{wt} \%)$ & $\Delta \sigma / \sigma$ & $l_{0}\left(\mathrm{~mm}^{-3} \mathrm{~s}^{-1}\right)$ \\
\hline NA-11 & 0.23 & $3.9 \times 10^{-2}$ & 0.13 & $1.3 \times 10^{11}$ \\
NA-11K & 0.37 & $1.1 \times 10^{-2}$ & 0.13 & $2.1 \times 10^{10}$ \\
Na-Bz & 2.0 & $3.4 \times 10^{-2}$ & 0.23 & $1.9 \times 10^{10}$ \\
het & - & - & 0.23 & $5.2 \times 10^{7}$ \\
\hline
\end{tabular}

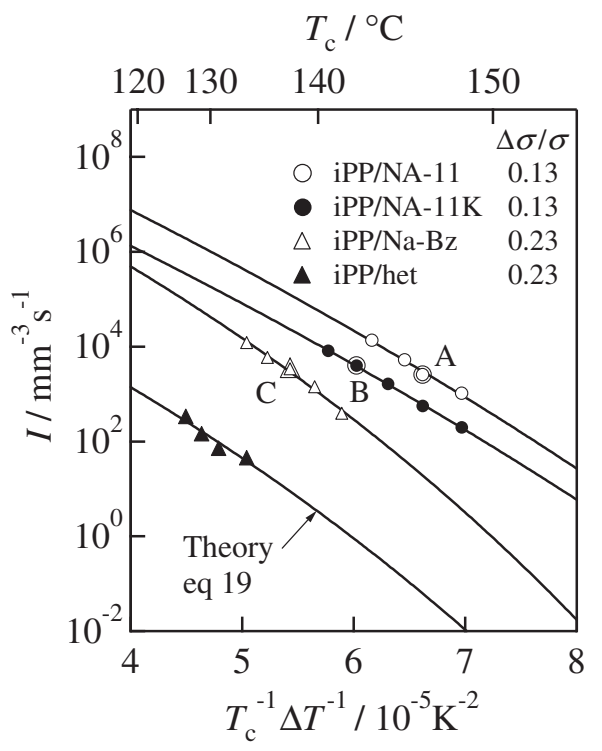

Figure 10. Plots of $/ \mathrm{s}$ of $\mathrm{iPP} / \mathrm{NA}-11\left(C_{\mathrm{NA}}=3.9 \times 10^{-2} \mathrm{wt} \%, a_{\mathrm{NA}}=0.23\right.$ $\mu \mathrm{m})$, iPP/NA-11K ( $\left.C_{\mathrm{NA}}=1.1 \times 10^{-2} \mathrm{wt} \%, \mathrm{a}_{\mathrm{NA}}=0.37 \mu \mathrm{m}\right)$, iPP/ $\mathrm{Na}-\mathrm{Bz}\left(C_{\mathrm{NA}}=3.4 \times 10^{-2}\right.$ wt $\left.\%, \mathrm{a}_{\mathrm{NA}}=2 \mu \mathrm{m}\right)$ and $\mathrm{PP} /$ het against $T^{-1} \Delta T^{-1}$. Upper axis indicates $T_{c}$. Eq 19 was fitted to the plots as parameters of $I_{0}$ and $\Delta \sigma / \sigma . \mathrm{A}, \mathrm{B}$ and $\mathrm{C}$ corresponds to those in Figure 9.

were observed in iPP/NA-11, iPP/NA-11K, and iPP/Na-Bz at higher $T_{\mathrm{c}}$ in comparison with $\mathrm{iPP} /$ het. The number of iPP crystals in iPP/NA-11 and iPP/NA-11K is larger than those in $\mathrm{iPP} / \mathrm{Na}-\mathrm{Bz}$ though $T_{\mathrm{c}}$ of $\mathrm{iPP} / \mathrm{NA}-11$ and $\mathrm{iPP} / \mathrm{NA}-11 \mathrm{~K}$ is higher than that of iPP/Na-Bz. Therefore the ability of $\mathrm{Na}-\mathrm{Bz}$ as NA should be worse than that of NA-11 and NA-11K.

\section{$\Delta G^{*}(\Delta \sigma)$ Dependence of $I$}

Typical plots of $v(t)$ against $t$ are shown in Figure 9. A, B and $\mathrm{C}$ indicates iPP/NA-11, iPP/NA-11K and iPP/Na-Bz, respectively. $I$ was obtained from the slope of $v(t)$ against $t$ (eq 27). Figure 9 shows that nearly the same $I$ s observed at $T_{\mathrm{c}}=146.6,142.7$ and $137.8^{\circ} \mathrm{C}$ for iPP/NA-11, iPP/NA-11K and $\mathrm{iPP} / \mathrm{Na}-\mathrm{Bz}$, respectively.

LogIs of iPP/NA-11, iPP/NA-11K, iPP/Na-Bz and iPP/het were plotted against $T_{\mathrm{c}}^{-1} \Delta T^{-1}$ in Figure 10. Upper horizontal axis indicates $T_{\mathrm{c}} \cdot \overline{a_{\mathrm{NA}}}$ and $C_{\mathrm{NA}}$ are shown in Table II. The plots were fitted by the theoretical curves given by eq 19 as parameters of $I_{0}$ and $\Delta \sigma$. For the fitting, the literature value of $\sigma$ and $\sigma_{\mathrm{e}}$ in Table I were used. ${ }^{9,10}$ All the curves show good fits to the plots. $\Delta \sigma / \sigma$ and $I_{0}$ obtained from the fitting are summarized in Table II. $\Delta \sigma / \sigma$ of NA- $11 \mathrm{~K}$ is the same as that 
(a)

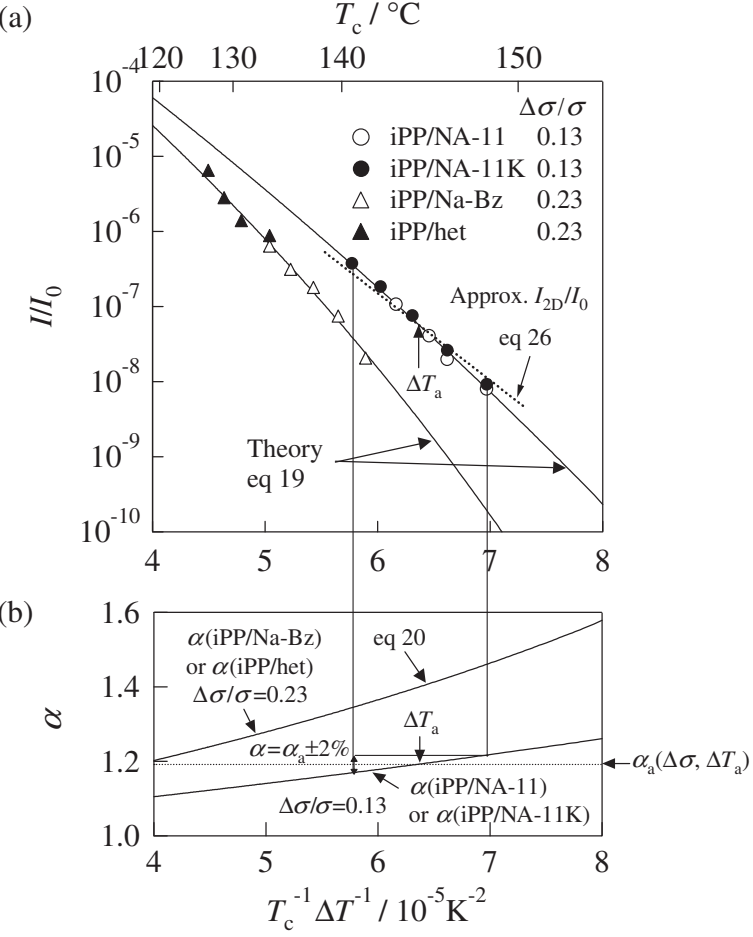

Figure 11. (a) Plots of $1 / \mathrm{I}_{0} \mathrm{~s}$ of iPP/NA-11, iPP/NA-11K, iPP/Na-Bz and iPP/het against $T_{\mathrm{c}}^{-1} \Delta T^{-1}$. Upper axis indicates $T_{\mathrm{c}}$. Solid curves show theoretical $I_{2 \mathrm{D}} / I_{0}$ given by eq 19 . Broken line shows approximated $l_{20} / l_{0}$ of iPP/NA-11K given by eq 26 . (b) Theoretical plots of $\alpha$ of iPP/NA-11, iPP/NA-11K, iPP/Na-Bz and $\mathrm{iPP} /$ het against $T_{\mathrm{c}}^{-1} \Delta T^{-1} \cdot \alpha_{\mathrm{a}}$ is the value of $\alpha$ at $\Delta T_{\mathrm{a}}$.

of NA-11 though degree of misfit between NA-11K and iPP crystals is better than that between NA-11 and iPP crystals for an azimuthal orientation (eq 13). Degree of misfit between NA$11 \mathrm{~K}$ and iPP crystals for the other azimuthal orientation could be larger than that between NA-11 and iPP crystals. $\Delta \sigma / \sigma$ of $\mathrm{Na}-\mathrm{Bz}$ is much larger than that of NA-11 and the same as that of some heterogeneity in $\mathrm{iPP} /$ het.

The plots and curves of $I / I_{0}$ against $T_{\mathrm{c}}^{-1} \Delta T^{-1}$ are shown in Figure 11a. As $I$ is divided by $I_{0}$, we can discuss $\Delta G^{*}$ dependence of $I$ irrespective of $I_{0}$. Upper horizontal axis indicates $T_{\mathrm{c}} . I / I_{0} \mathrm{~s}$ of $\mathrm{iPP} / \mathrm{NA}-11$ and $\mathrm{iPP} / \mathrm{NA}-11 \mathrm{~K}$ are the same. $I / I_{0}$ of iPP/NA-11 is $2 \times 10^{2}$ times for $\Delta T=30 \mathrm{~K}$ and 4 times for $\Delta T=50 \mathrm{~K}$ as large as that of $\mathrm{iPP} / \mathrm{Na}-\mathrm{Bz}$. Therefore $\Delta \sigma / \sigma$ is significantly important for acceleration of $I$. $I / I_{0}$ increased with decreasing $\Delta \sigma / \sigma$.

The curves of $\alpha$ of iPP/NA-11, iPP/NA-11K, iPP/Na-Bz, and $\mathrm{iPP} /$ het against $T_{\mathrm{c}}^{-1} \Delta T^{-1}$ are shown in Figure $11 \mathrm{~b}$. As $\Delta \sigma / \sigma$ of iPP/NA-11 and iPP/NA-11K are the same, $\alpha$ of iPP/ NA-11 and iPP/NA-11 is the same. $\alpha$ increases with increasing $\Delta \sigma / \sigma$. In the observed range of $T_{\mathrm{c}}^{-1} \Delta T^{-1}$ for iPP/NA-11K, $\alpha_{\mathrm{a}}$ given by eq 24 for $\Delta T_{\mathrm{a}}=37.5 \mathrm{~K}$ and the difference between $\alpha_{\mathrm{a}}$ and $\alpha$ for $\Delta T_{\mathrm{a}}=37.5 \mathrm{~K}$ are

$$
\alpha_{\mathrm{a}}=1.19 \text { and } \alpha=\alpha_{\mathrm{a}} \pm 2 \%,
$$

respectively. Therefore the approximation by eq 24 should be applicable. The dotted line in Figure 11a is the approximated line in eq 26 for iPP/NA-11K $\left(\alpha_{\mathrm{a}}=1.19\right)$. The dotted line was shifted to intersect with the curve of $I_{2 \mathrm{D}} / I_{0}$ of iPP/NA-11K at $\Delta T_{\mathrm{a}}$ by changing $I_{0}$. The approximated line rather agreed with the fitted line in the narrow range of $\Delta T$. We conclude that the analysis method by the approximation of eq 26 is efficiently to determine $\Delta \sigma$ of polymers in routine work.

\section{CONCLUSIONS}

1. Free energy for forming critical nucleus $\Delta G^{*}(\Delta \sigma)$ is essentially important for acceleration of nucleation where $\Delta \sigma$ is reflected in efficiency of epitaxy. $\Delta G^{*}(\Delta \sigma)$ dependence of nucleation rate $I$ was evaluated on the basis of nucleation theory. $I$ for the two dimensional nucleus $\left(I_{2 \mathrm{D}}\right)$ is given by

$$
\frac{I_{2 \mathrm{D}}(\Delta \sigma)}{I_{0}}=\exp \left(-\frac{\Delta G_{2 \mathrm{D}}^{*}}{k T_{\mathrm{c}}}\right)=\exp \left\{-\frac{\alpha(\Delta \sigma) \mathrm{B}}{T_{\mathrm{c}} \Delta T}\right\},
$$

where $I_{0}$ is prefactor, $k$ is Boltzmann constant $T_{\mathrm{c}}$ is crystallization temperature, $\alpha=\Delta g /(\Delta g-\Delta \sigma)$ where $\Delta g$ is free energy of fusion, $\mathrm{B}$ is constant, and $\Delta T$ is degree of supercooling. $I$ increased by 38 orders of magnitude for $\Delta T=30 \mathrm{~K}$ and 12 orders of magnitude for $\Delta T=50 \mathrm{~K}$ with decrease in $\Delta \sigma / \sigma$ from 1 to 0 where $\sigma$ is lateral surface free energy of nucleus. In the case $\Delta \sigma / \sigma<0.3, I$ increased by 6 orders of magnitude for $\Delta T=30 \mathrm{~K}$ and 60 times for $\Delta T=50 \mathrm{~K}$ with decreasing $\Delta \sigma / \sigma$ from 0.3 to $0 . I$ deeply depends on $\Delta \sigma / \sigma$. Therefore we conclude that $\Delta G^{*}(\Delta \sigma)$ is essentially important for acceleration of $I$ in heterogeneous nucleation.

2. The evaluation method of $\Delta \sigma$ was established. $\Delta \sigma$ and prefactor $I_{0}$ of isotactic polypropylene (iPP) mixed with three nucleating agents (NAs) were obtained from the fit of the above theoretical equation to the experimental plots of $I$ as parameters of $\Delta \sigma$ and $I_{0} . \Delta \sigma / \sigma$ of sodium 2,2'-methylene-bis-(4,6di-t-butylphenylene) phosphate (NA-11) and potassium $2,2^{\prime}$ methylene-bis-(4,6-di-t-butylphenylene)phosphate (NA-11K) were 0.13 though degree of misfit for an azimuthal orientation between NA-11K and iPP crystals is better than that between NA-11 and iPP crystals. In the case of sodium benzoate (Na$\mathrm{Bz}), \Delta \sigma / \sigma=0.23$ was obtained. $\Delta \sigma / \sigma$ of Na-Bz is larger than that of NA-11 or NA-11K. $I / I_{0}$ increased with decreasing $\Delta \sigma / \sigma$.

3. $I / I_{0}$ of iPP mixed with NA-11 is $2 \times 10^{2}$ times for $\Delta T=$ $30 \mathrm{~K}$ and 4 times for $\Delta T=50 \mathrm{~K}$ as large as that of iPP mixed with Na-Bz. Therefore $\Delta \sigma / \sigma$ is significantly important for acceleration of $I$ of polymers by NA.

\section{Appendix: $\Delta G^{*}$ of Lateral Growth}

It is well known in CNT that the lateral growth rate $V$ is mainly controlled by the secondary nucleation. $V$ is given by

$$
V=V_{0} \exp \left\{-\frac{\Delta G^{*}(2 n d)}{\gamma k T_{\mathrm{c}}}\right\}
$$

where $V_{0}$ is a prefactor, $\Delta G^{*}(2 \mathrm{nd})$ is free energy for forming critical nucleus $\Delta G^{*}$ of the secondary nucleus, and $\gamma=1$ or 3 are single or multi nucleation, respectively. ${ }^{15}$ As the secondary 
nucleus corresponds to the limit case of the heterogeneous 2D nucleus with $\Delta \sigma=0, \Delta G^{*}(2 \mathrm{nd})$ is given by

$$
\begin{aligned}
\Delta G^{*}(2 n d) & =\Delta G_{2 D}^{*}(\Delta \sigma=0) \\
& =\frac{4\left(\sigma \sigma_{e}\right)}{\Delta g}=\left\{\frac{4\left(\sigma \sigma_{\mathrm{e}}\right) T_{\mathrm{m}}^{0}}{\Delta h}\right\} \frac{1}{\Delta T} .
\end{aligned}
$$

Insertion of eq $\mathrm{A} \cdot 2$ into eq $\mathrm{A} \cdot 1$ gives

$$
\begin{aligned}
& \frac{V}{V_{0}}=\exp \left\{-\frac{\Delta G^{*}(2 n d)}{\gamma k T_{\mathrm{c}}}\right\}=\exp \left(-\frac{\mathrm{B}}{\gamma T_{\mathrm{c}} \Delta T}\right) \\
& \text { where } \mathrm{B}=\frac{4\left(\sigma \sigma_{\mathrm{e}}\right) T_{\mathrm{m}}^{0}}{k \Delta h} .
\end{aligned}
$$

$\left(\sigma \sigma_{\mathrm{e}}\right)$ can be obtained from the slope of the plots of $V$ against $T_{\mathrm{c}}^{-1} \Delta T^{-1}$, i.e., $-\mathrm{B} / \gamma$.

Received: May 14, 2008 Accepted: December 7, 2008 Published: January 28, 2009

\section{REFERENCES}

1. K. Okada, K. Watanabe, T. Urushihara, A. Toda, and M. Hikosaka, Polymer, 48, 401 (2007).
2. T. Urushihara, K. Okada, K. Watanabe, A. Toda, E. Tobita, N. Kawamoto, and M. Hikosaka, Polym. J., 39, 55 (2007).

3. R. Becker and W. Döring, Ann. Phys., 24, 719 (1935).

4. D. Turnbull and J. C. Fisher, J. Chem. Phys., 17, 71 (1949).

5. F. P. Price, in "Nucleation," A. C. Zettlemoyer, Ed., Marcel Dekker, New York, 1969, chap. 8, p 405.

6. Y. Takeda, in "Epitakisharu Seityono Mekanizumu (Mechanism of epitaxial growth)," K. Nakajima, ed., Kyoritsu Shuppan, Tokyo, 2002, chap. 5, p180.

7. S. Yoshimoto, T. Ueda, K. Yamanaka, A. Kawaguchi, E. Tobita, and T. Haruna, Polymer, 42, 9627 (2001).

8. M. Hikosaka and T. Seto, Polym. J., 5, 111 (1973).

9. E. J. Clark and J. D. Hoffmann, Macromolecules, 17, 878 (1984).

10. K. Yamada, M. Hikosaka, A. Toda, S. Yamazaki, and K. Tagashira, J. Macromol. Sci. Part B, Phys., 42, 733 (2003).

11. J. Brandrup, E. H. Immergut, D. R. Bloch, and E. A. Grulke, in "Polymer Handbook," 4th ed., John Wiley \& Sons, New York, 2003, $\mathrm{V} / 23$.

12. J. J. Janimak, S. Z. D. Cheng, and P. A. Giusti, Macromolecules, 24, 2253 (1991).

13. K. Yamada, M. Hikosaka, A. Toda, S. Yamazaki, and K. Tagashira, Macromolecles, 36, 4790 (2003).

14. K. Yamada, M. Hikosaka, A. Toda, S. Yamazaki, and K. Tagashira, Macromolecles, 36, 4802 (2003).

15. A. Ohkawa, in "Kessho-Seicho (Crystal Growth)," Shokabo Publishing, Tokyo, 1977, chap. 1, p 23. 